\title{
Equivalent post-buckling models for the flexural behaviour of steel connections
}

\author{
L. Simões da Silva ${ }^{a, *}$, Ana Girão Coelho ${ }^{b}$, Eliseu Lucena Neto ${ }^{\text {c }}$ \\ a Departamento de Engenharia Civil, Fac. de Ciencias e Tecnologia, Universidade de Coimbra, Polo II - Pinhal de Marrocos, 3030 \\ Coimbra Codex, Portugal \\ ${ }^{\mathrm{b}}$ Instituto Superior de Engenharia de Coimbra, 3030, Coimbra, Portugal \\ ' ITA, 12235-390, S. José dos Campos, SP, Brazil
}

Received 19 May 1999; accepted 8 December 1999

\begin{abstract}
Analytical solutions for the evaluation of the behaviour of steel connections are presented which are able to reproduce their full non-linear behaviour. Because usual models for the analysis of steel connections consist of translational springs and rigid links whereby the springs exhibit a non-linear force-deformation response, usually taken as a bi-linear approximation, they require an incremental non-linear analysis. Using a substitute elastic post-buckling model where each bi-linear spring is replaced by two equivalent elastic springs in the context of a post-buckling stability analysis using an energy formulation, closed-form solutions are obtained for a connection loaded in bending. Application to a beam-to-column welded connection using the component (spring) characterisation of code regulations yields the same results in terms of moment resistance and initial stiffness, being additionally able to trace the full unstiffening response. (c) 2000 Elsevier Science Ltd. All rights reserved.
\end{abstract}

Keywords: Steep; Joints; Bending; Component method; Nonlinear behaviour

\section{Introduction}

Currently, the evaluation of the behaviour of connections relies on the independent evaluation of strength, stiffness and ductility properties in the context of the socalled component method [1]. Although the first two, for a calibrated range of steel and composite connections, are covered by independent procedures that yield a moment resistance and an initial stiffness [2], the latter still remains quite unexplored, despite some recent attempts at providing a more quantitative guidance [3].

In spite of these advances in connection behaviour over the traditional approach of pinned or fully rigid response, no analytical procedure able to predict the full

\footnotetext{
* Corresponding author. Tel.: +351-239-797216; fax: +351239-797217.

E-mail address: luis_silva@gipac.pt (L. Simões da Silva).
}

non-linear moment-rotation curve of a joint, based on the load-deformation response of the contributing components is currently available. This paper presents analytical expressions for the evaluation of the response of steel connections based on equivalent post-buckling models.

\section{Equivalent models}

\subsection{Introduction}

The component method consists of idealising a connection as a mechanical model composed of translational springs and rigid links, whereby the springs (components) represent a specific part of a joint that, depending on the type of loading, make an identified contribution to one or more of its structural properties, as illustrated in Fig. 1 for a typical welded beamto-column steel connection. 
(a)

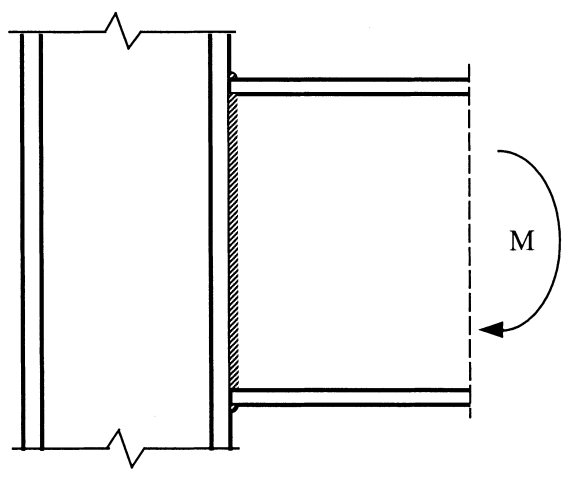

(b)

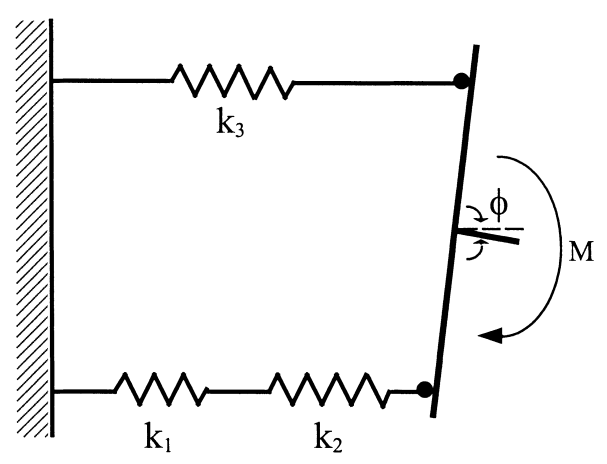

Fig. 1. Welded beam-to-column steel connection: (a) connection geometry and (b) mechanical model.

In the model of Fig. 1(b), spring $k_{2}$ represents the behaviour of the column web in compression [4], which is characterized by a non-linear curve with an initial elastic response and a subsequent stiffness reduction with limited ductility, as shown in Fig. 2(a). Similarly, springs $k_{1}$ and $k_{3}$ represent, respectively, the column web panel in shear [5] and in tension [6], which typically present the non-linear behaviour of Fig. 2(b), practically with unlimited ductility after an initial linear elastic response.

It is thus clear that a bi-linear force-displacement approximation adequately represents the component behaviour, involving the identification of four properties for each component, namely initial elastic stiffness $\left(k_{\mathrm{e}}\right)$, limit load $\left(F^{\mathrm{C}}\right)$, post-limit stiffness $\left(k_{\mathrm{p}}\right)$ and limit displacement $\left(\Delta^{f}\right)$, schematically shown in Fig. 3 , the yield displacement, $\Delta^{\mathrm{y}}$, being defined as $\left(\Delta^{\mathrm{y}}=F^{\mathrm{C}} / k_{\mathrm{e}}\right)$.

The springs and rigid links model of Fig. 1(b), with the assumed bi-linear behaviour of each spring (component) requires an incremental non-linear analysis when loaded in bending. Here, analytical moment-rotation curves are obtained using equivalent elastic models, replacing each bi-linear spring with an equivalent elastic system which yields the same response, in the context of a post-buckling elastic analysis. This approach was successfully employed in the context of the well-known Shanley model for the analysis of compressed columns in the elasto-plastic range [7]. (a)

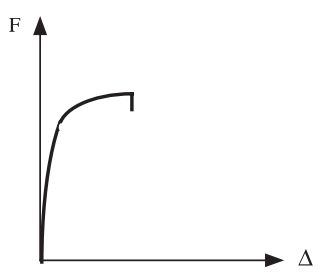

(b)

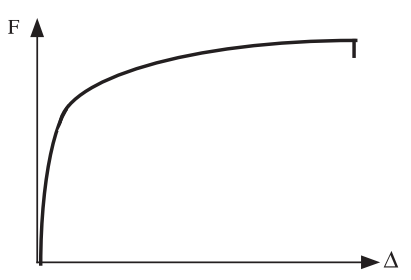

Fig. 2. Individual component behaviour: (a) column web in compression and (b) column web panel in shear or in tension.

\subsection{Non-linear model for equivalent elasto-plastic springs}

\subsubsection{Elasto-plastic spring in compression}

The basic building block of an equivalent model corresponds precisely to replacing each elasto-plastic (bi-linear) spring with an equivalent elastic system. Here, the two degree-of-freedom system of Fig. 4 is proposed, which consists of one elastic spring with stiffness $k_{\mathrm{e}}$ (linear elastic stiffness of component) and a second elastic spring with stiffness $k_{\mathrm{p}}$ (post-limit stiffness of component) and resistance $F^{\mathrm{C}}\left(=P^{\mathrm{B}} / 2\right)$ applied as a pre-compression, the degrees of freedom being defined as follows:

$\mathrm{Q}_{1}$ - total displacement,

$\mathrm{Q}_{2}$ - rotation of rigid links.

Clearly, this model exhibits distinct behaviour in tension and compression, the latter being of most interest because of the inherent bifurcational behaviour. Assuming a positive compressive load, and using an energy approach, the following total potential energy function is obtained:

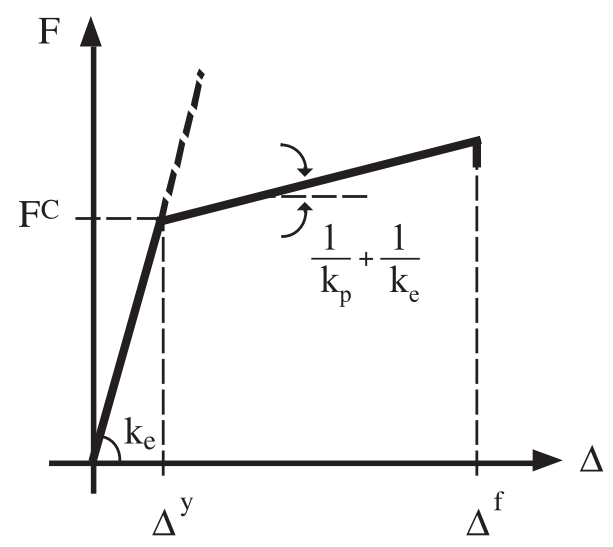

Fig. 3. Typical force-displacement diagram for generic component. 


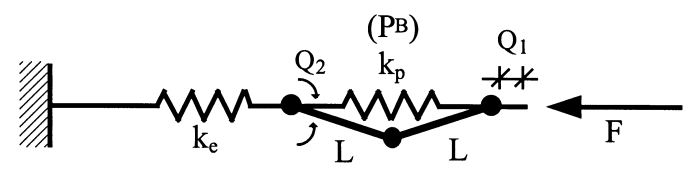

Fig. 4. Equivalent elastic system for elasto-plastic spring in compression.

$$
\begin{gathered}
V=\frac{1}{2} k_{\mathrm{e}}\left[Q_{1}-2 L\left(1-\cos Q_{2}\right)\right]^{2}+\frac{1}{2} k_{\mathrm{p}}\left[\frac{P^{\mathrm{B}}}{2 k_{\mathrm{p}}}\right. \\
\left.+2 L\left(1-\cos Q_{2}\right)\right]^{2}-F Q_{1} .
\end{gathered}
$$

Differentiation with respect to the various degrees of freedom yields the equilibrium equations

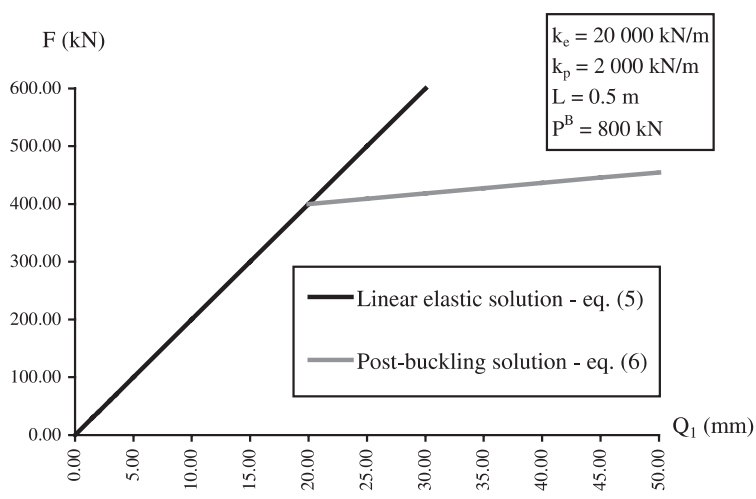

Fig. 5. Force-deformation solutions for an equivalent elastic system.

$$
\left\{\begin{array} { l } 
{ \frac { \partial V } { \partial Q _ { 1 } } = 0 } \\
{ \frac { \partial V } { \partial Q _ { 2 } } = 0 }
\end{array} \Longleftrightarrow \left\{\begin{array}{l}
k_{\mathrm{e}} Q_{1}-2 L k_{\mathrm{e}}\left(1-\cos Q_{2}\right)-F=0 \\
\operatorname{sen} Q_{2}\left[4 L^{2}\left(k_{\mathrm{e}}+k_{\mathrm{p}}\right)\left(1-\cos Q_{2}\right)-2 L Q_{1}+P^{\mathrm{B}}\right]=0
\end{array}\right.\right.
$$

giving

$Q_{1}=\frac{1}{k_{\mathrm{e}}}\left[2 L k_{\mathrm{e}}\left(1-\cos Q_{2}\right)+F\right]$,

which can be replaced in Eq. (1) to give

$$
\begin{aligned}
V\left(Q_{2}\right)= & 2 L^{2} k_{\mathrm{p}}\left(1-\cos Q_{2}\right)^{2}+P^{\mathrm{B}} L\left(1-\cos Q_{2}\right) \\
& -2 L F\left(1-\cos Q_{2}\right)+\frac{\left(P^{\mathrm{B}}\right)^{2}}{8 k_{\mathrm{p}}}-\frac{F^{2}}{2 k_{\mathrm{e}}} .
\end{aligned}
$$

Solution of Eq. (2) yields a trivial fundamental solution

$\left\{\begin{array}{l}Q_{2}=0 \\ F=k_{\mathrm{e}} Q_{1}\end{array}\right.$

and a post-buckling solution

$$
\left\{\begin{array}{l}
F=2 L k_{\mathrm{p}}\left(1-\cos Q_{2}\right)+\frac{p^{\mathrm{B}}}{2}, \\
F=\frac{k_{\mathrm{e}}}{k_{\mathrm{e}}+k_{\mathrm{p}}}\left(k_{\mathrm{p}} Q_{1}+\frac{P^{\mathrm{B}}}{2}\right) .
\end{array}\right.
$$

Differentiating Eq. (4) twice with respect to $Q_{2}$, evaluating along the fundamental path and equating to zero yields the critical load

$$
\begin{aligned}
\left.\frac{\mathrm{d}^{2} V}{\mathrm{~d} Q_{2}^{2}}\left(Q_{2}\right)\right|^{E} & =\left.\frac{\mathrm{d}^{2} V}{\mathrm{~d} Q_{2}^{2}}\right|_{Q_{2}=0} \\
& =4 L^{2} k_{\mathrm{p}}[\cos 0-\cos (0)]+L\left(P^{\mathrm{B}}-2 F\right) \cos 0 \\
& =L\left(P^{\mathrm{B}}-2 F\right)
\end{aligned}
$$

$$
F^{\mathrm{C}}=\frac{P^{\mathrm{B}}}{2} .
$$

The resulting force-deformation curves are shown in Fig. 5, clearly reproducing the bi-linear behaviour of the original elasto-plastic spring (component).

Under tensile loading, similar reasoning yields simply the linear elastic solution,

$F=k_{\mathrm{e}} Q_{1}$,

the bifurcational response being absent.

\subsubsection{Elasto-plastic spring in tension}

As seen in the previous section, the equivalent model of Fig. 4 is not able to reproduce a bi-linear response in tension. Such a situation requires the elastic system of Fig. 6, which, using a similar derivation and assuming $F$ to be positive in tension, yields the same equilibrium solutions of Eqs. (5), (6) and (8), and exhibits the required bi-linear response of the component in tension and a linear elastic behaviour when loaded in compression.

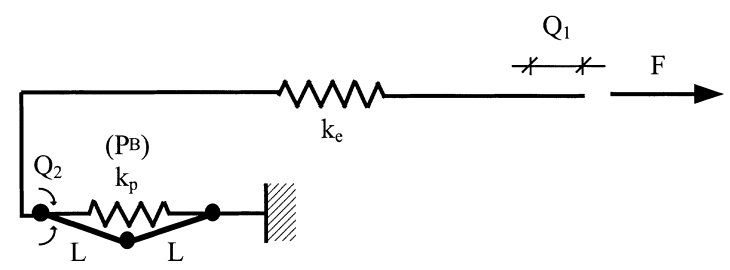

Fig. 6. Equivalent elastic system for an elasto-plastic spring in tension. 


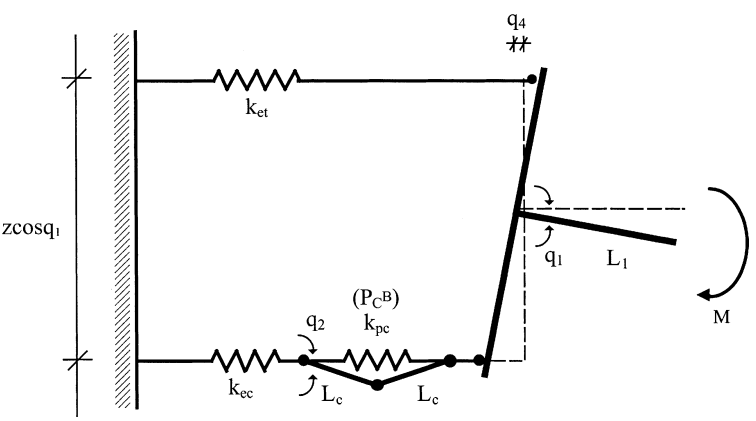

Fig. 7. Equivalent elastic model for a non-linear compression zone.

\subsection{Non-linear model for the shear and compression zones}

Having established a substitute model for one individual bi-linear spring, dealing with a steel connection requires introducing it in the model of Fig. 1(b). Starting, for simplicity, with an equivalent model where only one component in compression is assumed to reach the unstiffening load, the three degree-of-freedom model of Fig. 7 applies.

Besides the total rotation of the joint $\left(q_{1}\right)$ and the rotation of the rigid links $\left(q_{2}\right)$ already explained, a third degree-of-freedom ( $q_{4}$ - axial displacement of the connection) is required, because of the shift in neutral axis caused by asymmetries in spring stiffnesses between the tension and compression zones. Combining, for simplicity, the linear elastic stiffness of components 1 and 2 into one single equivalent elastic spring

$\frac{1}{k_{\mathrm{ec}}}=\frac{1}{k_{\mathrm{e} 1}}+\frac{1}{k_{\mathrm{e} 2}}$

the total potential energy function for this system can be obtained:

$$
\begin{gathered}
V=\frac{1}{2} k_{\mathrm{et}}\left(q_{4}-\frac{z}{2} \operatorname{sen} q_{1}\right)^{2}+\frac{1}{2} k_{\mathrm{ec}}\left[q_{4}+\frac{z}{2} \operatorname{sen} q_{1}\right. \\
\left.-2 L_{\mathrm{c}}\left(1-\cos q_{2}\right)\right]^{2}+\frac{1}{2} k_{\mathrm{pc}}\left[\frac{P_{\mathrm{C}}^{\mathrm{B}}}{2 k_{\mathrm{pc}}}\right. \\
\left.+2 L_{\mathrm{c}}\left(1-\cos q_{2}\right)\right]^{2}-M q_{1} .
\end{gathered}
$$

Eliminating $q_{4}$ as a passive coordinate through differentiation of Eq. (11) with respect to $q_{4}$ and equating to zero and substituting back into Eq. (11) yields, successively,

$$
\begin{aligned}
\frac{\partial V}{\partial q_{4}}=0 & \Longleftrightarrow q_{4} \\
= & \frac{1}{k_{\mathrm{ec}}+k_{\mathrm{et}}}\left[-\frac{z}{2}\left(k_{\mathrm{ec}}-k_{\mathrm{et}}\right) \operatorname{sen} q_{1}\right. \\
& \left.+2 L_{\mathrm{c}} k_{\mathrm{ec}}\left(1-\cos q_{2}\right)\right]
\end{aligned}
$$

$$
\begin{aligned}
V= & \frac{z^{2}}{2} \frac{k_{\mathrm{ec}} k_{\mathrm{et}}}{k_{\mathrm{ec}}+k_{\mathrm{et}}} \operatorname{sen}^{2} q_{1}+2 L_{\mathrm{c}}^{2}\left(k_{\mathrm{pc}}+\frac{k_{\mathrm{ec}} k_{\mathrm{et}}}{k_{\mathrm{ec}}+k_{\mathrm{et}}}\right) \\
& \times\left(1-\cos q_{2}\right)^{2}-\left(\frac{2 z L_{\mathrm{c}} k_{\mathrm{ec}} k_{\mathrm{et}}}{k_{\mathrm{ec}}+k_{\mathrm{et}}} \operatorname{sen} q_{1}-P_{\mathrm{C}}^{\mathrm{B}} L_{\mathrm{c}}\right) \\
& \times\left(1-\cos q_{2}\right)+\frac{\left(P_{\mathrm{C}}^{\mathrm{B}}\right)^{2}}{8 k_{\mathrm{pc}}}-M q_{1} .
\end{aligned}
$$

Differentiation with respect to $q_{1}$ and $q_{2}$ gives the equilibrium equations of the system:

$$
\begin{aligned}
\frac{\partial V}{\partial q_{1}}= & z^{2} \frac{k_{\mathrm{ec}} k_{\mathrm{et}}}{k_{\mathrm{ec}}+k_{\mathrm{et}}} \operatorname{sen} q_{1} \cos q_{1} \\
& -\frac{2 z L_{\mathrm{c}} k_{\mathrm{ec}} k_{\mathrm{et}}}{k_{\mathrm{ec}}+k_{\mathrm{et}}} \cos q_{1}\left(1-\cos q_{2}\right)-M=0 \\
\frac{\partial V}{\partial q_{2}}= & 4 L_{\mathrm{c}}^{2}\left(\frac{k_{\mathrm{pc}}+k_{\mathrm{ec}} k_{\mathrm{et}}}{k_{\mathrm{ec}}+k_{\mathrm{et}}}\right) \operatorname{sen} q_{2}\left(1-\cos q_{2}\right) \\
& -\left(\frac{2 z L_{\mathrm{c}} k_{\mathrm{ec}} k_{\mathrm{et}}}{k_{\mathrm{ec}}+k_{\mathrm{et}}} \operatorname{sen} q_{1}-P_{\mathrm{C}}^{\mathrm{B}} L_{\mathrm{c}}\right) \operatorname{sen} q_{2}=0
\end{aligned}
$$

which correspond to a fundamental solution

$\left\{\begin{array}{l}M=\frac{z^{2}}{2} \frac{k_{\mathrm{ec}} k_{\mathrm{et}}}{k_{\mathrm{ec}}+k_{\mathrm{et}}} \operatorname{sen}\left(2 q_{1}\right), \\ q_{2}=0\end{array}\right.$

and a coupled solution

$$
\left\{\begin{array}{l}
M=\frac{z k_{\mathrm{ec}} k_{\mathrm{et}}}{k_{\mathrm{ec}}+k_{\mathrm{et}}}\left[z \operatorname{sen} q_{1}-\frac{2 z k_{\mathrm{ec}} k_{\mathrm{et}} \operatorname{sen} q_{1}-P_{\mathrm{C}}^{\mathrm{B}}\left(k_{\mathrm{ec}}+k_{\mathrm{et}}\right)}{2\left[k_{\mathrm{pc}}\left(k_{\mathrm{ec}}+k_{\mathrm{et}}\right)+k_{\mathrm{ec}} k_{\mathrm{et}}\right]}\right] \cos q_{1} \\
1-\cos q_{2}=\frac{2 z k_{\mathrm{ec}} k_{\mathrm{et}} \operatorname{sen} q_{1}-P_{\mathrm{C}}^{\mathrm{B}}\left(k_{\mathrm{ec}}+k_{\mathrm{et}}\right)}{4 L_{\mathrm{c}}\left[k_{\mathrm{pc}}\left(k_{\mathrm{ec}}+k_{\mathrm{et}}\right)+k_{\mathrm{ec}} k_{\mathrm{et}}\right]}
\end{array}\right.
$$

\subsection{Non-linear model for the tension zone}

Likewise, should the tensile component present the lowest critical value for the limit load, the equivalent

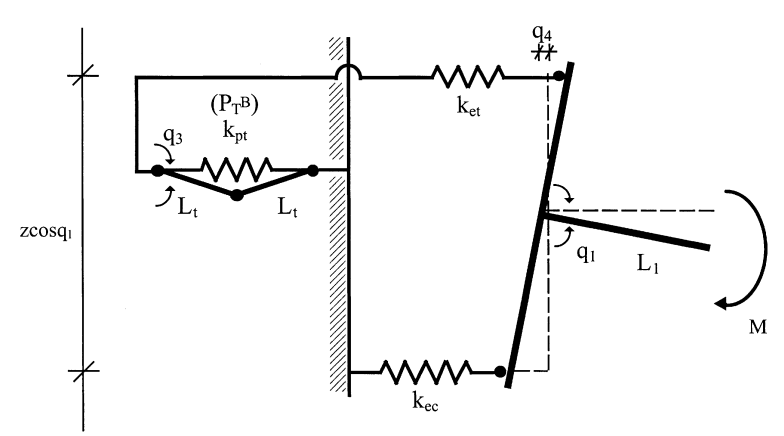

Fig. 8. Equivalent elastic model for a non-linear tensile zone. 


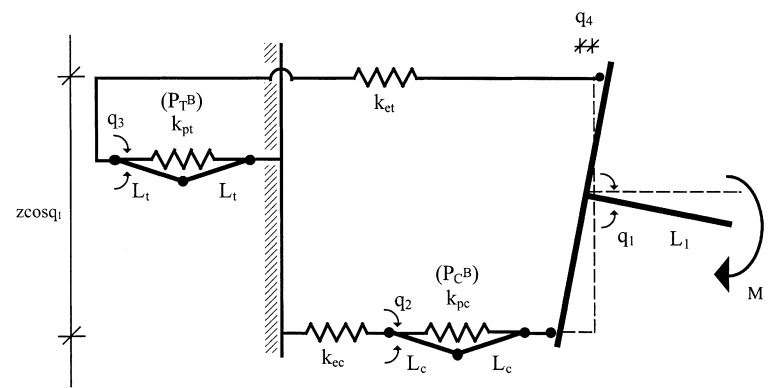

Fig. 9. General equivalent elastic model.

model of Fig. 8 applies, the same degrees of freedom being valid.

\subsection{General non-linear model of connection}

Finally, a more general model can be proposed which caters for the bi-linear component behaviour both in the tensile and compressive zones. From the model of Fig. 9, using the same procedure as above yields in succession

$$
\begin{aligned}
& V=\frac{1}{2} k_{\mathrm{ec}}\left[q_{4}+\frac{z}{2} \operatorname{sen} q_{1}-2 L_{\mathrm{c}}\left(1-\cos q_{2}\right)\right]^{2} \\
& +\frac{1}{2} k_{\mathrm{et}}\left[q_{4}-\frac{z}{2} \operatorname{sen} q_{1}+2 L_{\mathrm{t}}\left(1-\cos q_{3}\right)\right]^{2} \\
& +\frac{1}{2} k_{\mathrm{pc}}\left[\frac{P_{\mathrm{C}}^{\mathrm{B}}}{2 k_{\mathrm{pc}}}+2 L_{\mathrm{c}}\left(1-\cos q_{2}\right)\right]^{2} \\
& +\frac{1}{2} k_{\mathrm{pt}}\left[\frac{P_{\mathrm{T}}^{\mathrm{B}}}{2 k_{\mathrm{pt}}}+2 L_{\mathrm{t}}\left(1-\cos q_{3}\right)\right]^{2}-M_{q_{1}}, \\
& \frac{\partial V}{\partial q_{4}}=0 \Longleftrightarrow q_{4} \\
& =\frac{1}{k_{\mathrm{ec}}+k_{\mathrm{et}}}\left[-\frac{z}{2}\left(k_{\mathrm{ec}}-k_{\mathrm{et}}\right) \operatorname{sen} q_{1}\right. \\
& \left.+2 L_{\mathrm{c}} k_{\mathrm{ec}}\left(1-\cos q_{2}\right)-2 L_{\mathrm{t}} k_{\mathrm{et}}\left(1-\cos q_{3}\right)\right], \\
& V=\frac{z^{2}}{2} \frac{k_{\mathrm{ec}} k_{\mathrm{et}}}{k_{\mathrm{ec}}+k_{\mathrm{et}}} \operatorname{sen}^{2} q_{1}+2 L_{\mathrm{c}}^{2}\left(k_{\mathrm{pc}}+\frac{k_{\mathrm{ec}} k_{\mathrm{et}}}{k_{\mathrm{ec}}+k_{\mathrm{et}}}\right) \\
& \times\left(1-\cos q_{2}\right)^{2}+2 L_{\mathrm{t}}^{2}\left(k_{\mathrm{pt}}+\frac{k_{\mathrm{ec}} k_{\mathrm{et}}}{k_{\mathrm{ec}}+k_{\mathrm{et}}}\right) \\
& \times\left(1-\cos q_{3}\right)^{2}-\left(\frac{2 z l_{\mathrm{c}} k_{\mathrm{ec}} k_{\mathrm{et}}}{k_{\mathrm{ec}}+k_{\mathrm{et}}} \operatorname{sen} q_{1}-P_{\mathrm{C}}^{\mathrm{B}} L_{\mathrm{c}}\right) \\
& \times\left(1-\cos q_{2}\right)-\left(\frac{2 z L_{\mathrm{t}} k_{\mathrm{ec}} k_{\mathrm{et}}}{k_{\mathrm{ec}}+k_{\mathrm{et}}} \operatorname{sen} q_{1}-P_{\mathrm{T}}^{\mathrm{B}} L_{\mathrm{t}}\right) \\
& \times\left(1-\cos q_{3}\right)+4 L_{\mathrm{c}} L_{\mathrm{t}} \frac{k_{\mathrm{ec}} k_{\mathrm{et}}}{k_{\mathrm{ec}}+k_{\mathrm{et}}}\left(1-\cos q_{2}\right) \\
& \times\left(1-\cos q_{3}\right)+\frac{\left(P_{\mathrm{C}}^{\mathrm{B}}\right)^{2}}{8 k_{\mathrm{pc}}}+\frac{\left(P_{\mathrm{T}}^{\mathrm{B}}\right)^{2}}{8 k_{\mathrm{pt}}}-M_{q_{1}},
\end{aligned}
$$

$$
\begin{aligned}
\frac{\partial V}{\partial q_{1}}= & z^{2} \frac{k_{\mathrm{ec}} k_{\mathrm{et}}}{k_{\mathrm{ec}}+k_{\mathrm{et}}} \operatorname{sen} q_{1} \cos q_{1}-\frac{2 z L_{\mathrm{c}} k_{\mathrm{ec}} k_{\mathrm{et}}}{k_{\mathrm{ec}}+k_{\mathrm{et}}} \cos q_{1}\left(1-\cos q_{2}\right) \\
& -\frac{2 z L_{\mathrm{t}} k_{\mathrm{ec}} k_{\mathrm{et}}}{k_{\mathrm{ec}}+k_{\mathrm{et}}} \cos q_{1}\left(1-\cos q_{3}\right)-M=0 \\
\frac{\partial V}{\partial q_{2}}= & 4 L_{\mathrm{c}}^{2}\left(k_{\mathrm{ec}}+\frac{k_{\mathrm{ec}} k_{\mathrm{et}}}{k_{\mathrm{ec}}+k_{\mathrm{et}}}\right) \operatorname{sen} q_{2}\left(1-\cos q_{2}\right) \\
& -\left(\frac{2 z L_{\mathrm{c}} k_{\mathrm{ec}} k_{\mathrm{et}}}{k_{\mathrm{ec}}+k_{\mathrm{et}}} \operatorname{sen} q_{1}-P_{\mathrm{C}}^{\mathrm{B}} L_{\mathrm{c}}\right) \operatorname{sen} q_{2}
\end{aligned}
$$$$
+4 L_{\mathrm{c}} L_{\mathrm{t}} \frac{k_{\mathrm{ec}} k_{\mathrm{et}}}{k_{\mathrm{ec}}+k_{\mathrm{et}}} \operatorname{sen} q_{2}\left(1-\cos q_{3}\right)
$$$$
=0 \text {, }
$$

$$
\begin{aligned}
\frac{\partial V}{\partial q_{3}}= & 4 L_{\mathrm{t}}^{2}\left(k_{\mathrm{pt}}+\frac{k_{\mathrm{ec}} k_{\mathrm{et}}}{k_{\mathrm{ec}}+k_{\mathrm{et}}}\right) \operatorname{sen} q_{3}\left(1-\cos q_{3}\right) \\
& -\left(\frac{2 z L_{\mathrm{t}} k_{\mathrm{ec}} k_{\mathrm{et}}}{k_{\mathrm{ec}}+k_{\mathrm{et}}} \operatorname{sen} q_{1}-P_{\mathrm{T}}^{\mathrm{B}} L_{\mathrm{t}}\right) \operatorname{sen} q_{3} \\
& +4 L_{\mathrm{c}} L_{\mathrm{t}} \frac{k_{\mathrm{ec}} k_{\mathrm{et}}}{k_{\mathrm{ec}}+k_{\mathrm{et}}}\left(1-\cos q_{2}\right) \operatorname{sen} q_{3} \\
= & 0
\end{aligned}
$$

and the following solutions:

(i) Fundamental solution

$$
\left\{\begin{array}{l}
M=\frac{z^{2} k_{\mathrm{ec}} k_{\mathrm{et}}}{2\left(k_{\mathrm{ec}}+k_{\mathrm{et}}\right)} \operatorname{sen}\left(2 q_{1}\right), \\
q_{2}=0 \\
q_{3}=0
\end{array}\right.
$$

(ii) Non-linear solution in $q_{2}$

$$
\left\{\begin{array}{l}
M=\frac{z k_{\mathrm{ec}} k_{\mathrm{et}}}{k_{\mathrm{ec}}+k_{\mathrm{et}}}\left[z \operatorname{sen} q_{1}-\frac{2 z k_{\mathrm{ec}} k_{\mathrm{et}} \operatorname{sen} q_{1}-P_{\mathrm{C}}^{\mathrm{B}}\left(k_{\mathrm{ec}}+k_{\mathrm{et}}\right)}{2\left[k_{\mathrm{pc}}\left(k_{\mathrm{ec}}+k_{\mathrm{et}}\right)+k_{\mathrm{ec}} k_{\mathrm{et}}\right]}\right] \cos q_{1}, \\
1-\cos q_{2}=\frac{2 z k_{\mathrm{ec}} k_{\mathrm{et}} \operatorname{sen} q_{1}-P_{\mathrm{C}}^{\mathrm{B}}\left(k_{\mathrm{ec}}+k_{\mathrm{et}}\right)}{4 L_{\mathrm{c}}\left[k_{\mathrm{pc}}\left(k_{\mathrm{ec}}+k_{\mathrm{et}}\right)+k_{\mathrm{ec}} k_{\mathrm{et}}\right]}, \\
q_{3}=0 .
\end{array}\right.
$$

(iii) Non-linear solution in $q_{3}$

$$
\left\{\begin{array}{l}
M=\frac{z k_{\mathrm{ec}} k_{\mathrm{et}}}{k_{\mathrm{ec}}+k_{\mathrm{et}}}\left[z \operatorname{sen} q_{1}-\frac{2 z k_{\mathrm{ec}} k_{\mathrm{et}} \operatorname{sen} q_{1}-P_{\mathrm{T}}^{\mathrm{B}}\left(k_{\mathrm{ec}}+k_{\mathrm{et}}\right)}{2\left[k_{\mathrm{pt}}\left(k_{\mathrm{ec}}+k_{\mathrm{et}}\right)+k_{\mathrm{ec}} k_{\mathrm{et}}\right]}\right] \cos q_{1}, \\
q_{2}=0 \\
1-\cos q_{3}=\frac{2 z k_{\mathrm{ec}} k_{\mathrm{et}} \operatorname{sen} q_{1}-P_{\mathrm{T}}^{\mathrm{B}}\left(k_{\mathrm{ec}}+k_{\mathrm{et}}\right)}{4 L_{\mathrm{t}}\left[k_{\mathrm{pt}}\left(k_{\mathrm{ec}}+k_{\mathrm{et}}\right)+k_{\mathrm{ec}} k_{\mathrm{et}}\right]} .
\end{array}\right.
$$


(iv) Non-linear solution in $q_{2}$ and $q_{3}$

$$
\left\{\begin{array}{l}
M=\frac{z k_{\mathrm{ec}} k_{\mathrm{et}}}{k_{\mathrm{ec}}+k_{\mathrm{et}}}\left[z \operatorname{sen} q_{1}-2 L_{\mathrm{c}}\left(1-\cos q_{2}\right)-2 L_{\mathrm{t}}\left(1-\cos q_{3}\right)\right] \cos q_{1}, \\
1-\cos q_{2}=\frac{2 z k_{\mathrm{ec}} k_{\mathrm{et}} \operatorname{sen} q_{1}-P_{\mathrm{C}}^{\mathrm{B}}\left(k_{\mathrm{ec}}+k_{\mathrm{et}}\right)-4 L_{\mathrm{t}} k_{\mathrm{ec}} k_{\mathrm{et}}\left(1-\cos q_{3}\right)}{4 L_{\mathrm{c}}\left[k_{\mathrm{pc}}\left(k_{\mathrm{ec}}+k_{\mathrm{et}}\right)+k_{\mathrm{ec}} k_{\mathrm{et}}\right]} \\
1-\cos q_{3}=\frac{2 z k_{\mathrm{ec}} k_{\mathrm{et}} k_{\mathrm{pc}} \operatorname{sen} q_{1}-k_{\mathrm{ec}} k_{\mathrm{et}}\left(P_{\mathrm{T}}^{\mathrm{B}}-P_{\mathrm{C}}^{\mathrm{B}}\right)-P_{\mathrm{T}}^{B}\left(k_{\mathrm{ec}}+k_{\mathrm{et}}\right) k_{\mathrm{pc}}}{4 L_{\mathrm{t}}\left[k_{\mathrm{ec}} k_{\mathrm{et}}\left(k_{\mathrm{pc}}+k_{\mathrm{pt}}\right)+\left(k_{\mathrm{ec}}+k_{\mathrm{et}}\right) k_{\mathrm{pc}} k_{\mathrm{pt}}\right]} .
\end{array}\right.
$$

\section{Application to beam-to-column welded connections}

\subsection{Component characterisation}

In order to illustrate the application of the equivalent elastic models, one connection configuration was chosen from the database SERICON II (Klein 105.010) [8], corresponding to a welded beam-to-column steel connection, described in Fig. 1, which was tested by Klein at the University of Innsbruck in 1985.

As described above, the first step consists in establishing the components properties, initial stiffness $k_{\mathrm{i}}$, resistance $F_{\mathrm{i}}^{\mathrm{C}}$, post-limit stiffness $k_{\mathrm{pi}}$ and maximum displacement $\Delta_{\mathrm{i}}^{f}$. From Fig. 1, three contributing components are identified, namely:

(1) column web in shear,

(2) column web in compression,

(3) column web in tension.

Using the specifications of the revised Annex $\mathrm{J}$ of Eurocode 3 [2], the results shown in Table 1 were obtained for the initial stiffness of each component, where $k_{1}, k_{2}$ and $k_{3}$ denote the initial stiffness of components 1 , 2 and 3, respectively, non-dimensionalised with respect to Young's modulus. It is noted that the remaining quantities are described in Ref. [2]. From Eq. (10),

$k_{\mathrm{ec}}=\frac{E}{\frac{1}{k_{1}}+\frac{1}{k_{2}}}=0.3818 \times 10^{6} \mathrm{kN} / \mathrm{m} \quad$ and

$k_{\mathrm{et}}=k_{3} \times E=1.6666 \times 10^{6} \mathrm{kN} / \mathrm{m}$.

For the resistance (limit load) of each component, again following the revised Annex $\mathbf{J}$ [2], corresponding results are shown in Table 2.
It is noted that no ductility limits were imposed on each component because of lack of data. Also, the postlimit stiffness is currently not covered by code regulations.

\subsection{Numerical model}

To confirm the results from the analytical model, a numerical model was implemented using the non-linear finite element system LUSAS [9], shown in Fig. 10 and initially analysed using linear elastic properties for the springs (corresponding to initial stiffness) and subsequently analysed with bi-linear properties for the springs.

The definition of the finite element model is summarised in Table 3.

\subsection{Results}

Having established in Table 2 that the critical component was the column web in shear, an initial comparison was performed using only a bi-linear approximation for this component, the remaining being kept linear elastic. Table 4 and Fig. 11 show the corresponding results, values for the post-limit stiffness $k_{\mathrm{pc}}$ being chosen as zero to match the EC3 prediction. Next, a second comparison was made with all components as bi-linear springs, shown in Fig. 12.

It is clear that the numerical and analytical results are similar, and in agreement with the initial stiffness predictions of EC3. It is interesting to note in Fig. 12, the various equilibrium paths corresponding to the various components reaching their resistance. It should be noted

Table 1

Initial stiffness of each component

\begin{tabular}{lll}
\hline Column web in shear (J.4.2 (1)) & Column web in compression (J.4.2 (2)) & Column web in tension (J.4.2 (3)) \\
\hline$k_{1}=0.38 A_{\mathrm{vc}} / \beta z$ & $k_{2}=0.7 b_{\text {eff, }, \mathrm{wc}, t_{\mathrm{wc}} / d_{\mathrm{c}}}$ & $k_{3}=0.7 b_{\mathrm{eff}, \mathrm{t}, \mathrm{wc}} t_{\mathrm{wc}} / d_{\mathrm{c}}$ \\
$A_{\mathrm{vc}}=1308 \mathrm{~mm}^{2}$ & $b_{\mathrm{eff}, \mathrm{c}, \mathrm{wc}}=149.0 \mathrm{~mm}$ & $b_{\mathrm{eff}, \mathrm{t}, \mathrm{wc}}=149.0 \mathrm{~mm}$ \\
$z=h_{\mathrm{b}}-t_{\mathrm{f}, \mathrm{b}}=210.8 \mathrm{~mm}$ & $d_{\mathrm{c}}=92 \mathrm{~mm}$ & $d_{\mathrm{c}}=92 \mathrm{~mm}$ \\
$\beta \equiv \beta_{1}$ & & \\
Table J.4 $\rightarrow \beta \approx 1$ & & \\
$k_{1}=0.38 \times 1308 / 1 \times 210.8=2.358 \mathrm{~mm}$ & $k_{2}=0.7 \times 149.0 \times 7 / 92=7.936 \mathrm{~mm}$ & $k_{3}=0.7 \times 149.0 \times 7 / 92=7.936 \mathrm{~mm}$ \\
\hline
\end{tabular}


Table 2

Resistance of each component

\begin{tabular}{|c|c|c|}
\hline Column web in shear & Column web in compression & Column web in tension \\
\hline $\begin{array}{l}\mathrm{J} .3 .5 .1(1) V_{\mathrm{wd}, \mathrm{Rd}} \\
=0.9 f_{\mathrm{y}, \mathrm{wc}} A_{\mathrm{vc}} / \sqrt{3} \gamma_{M 0} \\
f_{\mathrm{y}, \mathrm{wc}}=275 \mathrm{MPa} \\
A_{\mathrm{vc}}=1308 \mathrm{~mm}^{2} \\
\gamma_{M 0}=1.1 \\
V_{\mathrm{wd}, \mathrm{Rd}}=170 \mathrm{kN}\end{array}$ & $\begin{array}{l}\text { (1) } F_{\mathrm{c}, \mathrm{wc}, \mathrm{Rd}}=\omega b_{\mathrm{eff}, \mathrm{c}, \mathrm{wc}} t_{\mathrm{wc}} f_{\mathrm{y}, \mathrm{wc}} / \gamma_{M 0} \\
\text { but } F_{\mathrm{cc}, \mathrm{wc}, \mathrm{Rd}} \leqslant \omega \rho b_{\mathrm{eff}, \mathrm{c}, \mathrm{wc}} t_{\mathrm{wc}} f_{\mathrm{y}, \mathrm{wc}} / \gamma_{\mathrm{M} 1} \\
\text { (3) } \bar{\lambda}_{\mathrm{p}}=0.932 \sqrt{b_{\mathrm{eff}, \mathrm{c}, \mathrm{wc}} d_{\mathrm{wc}} f_{\mathrm{y}, \mathrm{wc}} / E t_{\mathrm{wc}}^{2}} \\
b_{\mathrm{eff}, \mathrm{c}, \mathrm{wc}}=149.0 \mathrm{~mm} \\
d_{\mathrm{c}}=92 \mathrm{~mm} \\
f_{\mathrm{y}, \mathrm{wc}}=275 \mathrm{MPa} \\
E=210 \mathrm{GPa} \\
\bar{\lambda}_{\mathrm{p}}=0.932 \sqrt{149 \times 92 \times 275 / 210 \times 10^{3} \times 7^{2}}=0.56 \\
(2) \bar{\lambda}_{\mathrm{p}}=0.56<0.673 \Rightarrow \rho=1.0 \\
(4) \beta \equiv \beta_{1} \text { Table J.4 } \rightarrow \beta \approx 1 \\
\text { Table J.5 } \rightarrow \beta=1 \Rightarrow \omega=\omega_{1}=\frac{1}{\sqrt{1+1.3\left(b_{\mathrm{eff}, \mathrm{c}, \mathrm{wc}} t_{\mathrm{wc}} / A_{\mathrm{vc}}\right)^{2}}}=0.74 \\
\gamma_{M 0}=\gamma_{M 1}=1.1 \\
F_{\mathrm{c}, \mathrm{wc}, \mathrm{Rd}}=193 \mathrm{kN}\end{array}$ & $\begin{array}{l}\mathrm{J} .3 .5 .3 \\
(1) F_{\mathrm{t}, \mathrm{wc}, \mathrm{Rd}}=\omega b_{\mathrm{eff}, \mathrm{t}, \mathrm{wc}} t_{\mathrm{wc}} f_{\mathrm{y}, \mathrm{wc}} / \gamma_{M 0} \\
b_{\mathrm{eff}, \mathrm{c}, \mathrm{wc}}=149.0 \mathrm{~mm} \\
f_{\mathrm{y}, \mathrm{wc}}=275 \mathrm{MPa} \\
\beta \equiv \beta_{1} \text { Table J.4 } \rightarrow \beta \approx 1 \\
\text { Table J.5 } \rightarrow \beta=1 \Rightarrow \omega \\
=\omega_{1}=\frac{1}{\sqrt{1+1.3\left(b_{\mathrm{eff}, \mathrm{c}, \mathrm{wc}} t_{\mathrm{wc}} / A_{\mathrm{vc}}\right)^{2}}}=0.74 \\
\gamma_{M 0}=\gamma_{M 1}=1.1 \\
F_{\mathrm{t}, \mathrm{wc}, \mathrm{Rd}}=193 \mathrm{kN}\end{array}$ \\
\hline
\end{tabular}

Without safety coefficients

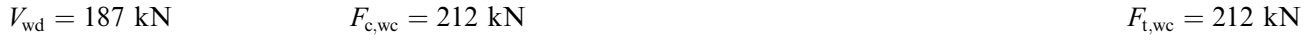

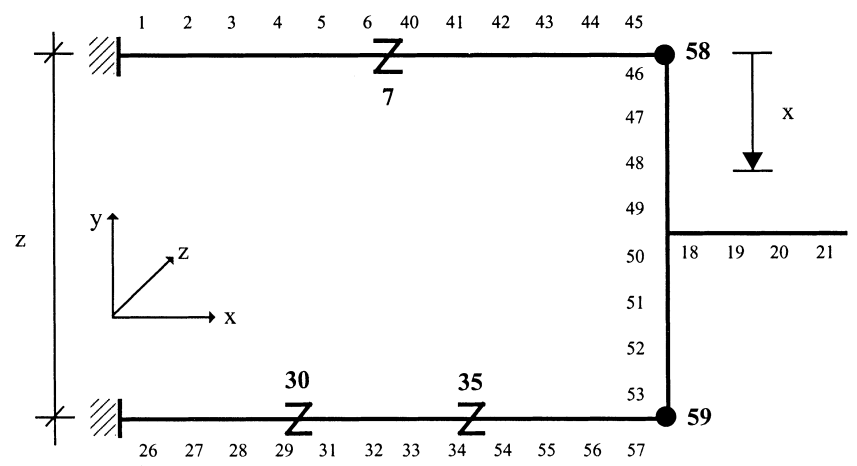

Element numbers

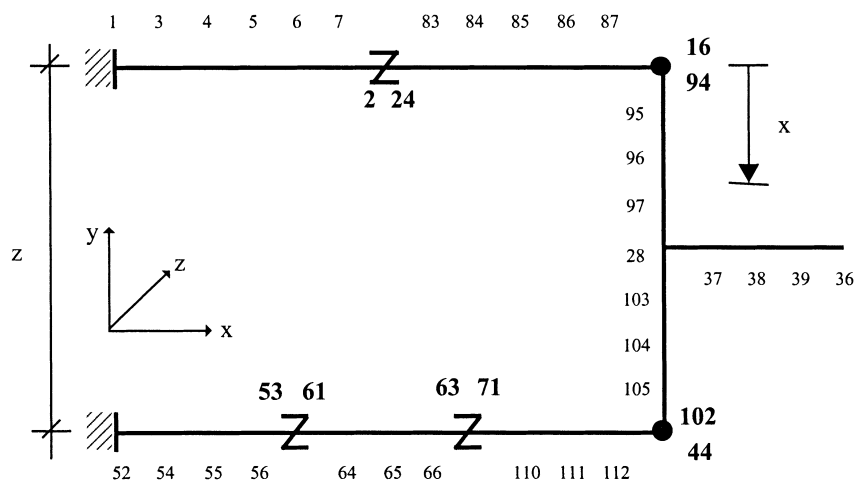

Node numbers

Z Joint symbol

Fig. 10. Finite element model. 
Table 3

Numerical model

\begin{tabular}{|c|c|c|c|c|}
\hline \multirow[t]{2}{*}{ Rigid links } & \multicolumn{3}{|l|}{ Joint elements (Component) } & \multirow[t]{2}{*}{ Pins } \\
\hline & Column web in shear & Column web in compression & Column web in tension & \\
\hline Finite element & BM3 & JPH3 & & \\
\hline Geometric properties & $\begin{array}{l}\text { Thin beam } A=100 \mathrm{~m}^{2}, \\
I_{\mathrm{x}}=I_{\mathrm{y}}=8.3333 \times 10^{3} \mathrm{~m}^{4}\end{array}$ & Eccentricity: $e=0$ & & \\
\hline Material & Elastic, isotropic & Joint (stiffness, 3DOF) & & \\
\hline \multirow[t]{3}{*}{$\begin{array}{l}E=210 \times 10^{6} \mathrm{kPa} \\
v=0.3 \\
\rho=7.850 \text { ton } / \mathrm{m}^{3}\end{array}$} & $\begin{aligned} k_{\mathrm{ex}} & =0.49518 \times 10^{6} \\
k_{\mathrm{ey}} & =0.1 \times 10^{20} \\
k_{\mathrm{e} \theta} & =0.1 \times 10^{20}\end{aligned}$ & $\begin{aligned} k_{\mathrm{ex}} & =1.6666 \times 10^{6} \\
k_{\mathrm{ey}} & =0.1 \times 10^{20} \\
k_{\mathrm{e} \theta} & =0.1 \times 10^{20}\end{aligned}$ & $\begin{aligned} k_{\mathrm{ex}} & =1.6666 \times 10^{6} \\
k_{\mathrm{ey}} & =0.1 \times 10^{20} \\
k_{\mathrm{e} \theta} & =0.1 \times 10^{20}\end{aligned}$ & $\begin{array}{l}k_{\text {ex }}=0.1 \times 10^{21} \\
k_{\text {ey }}=0.1 \times 10^{21} \\
k_{\text {e } \theta}=0.1 \times 10^{-19}\end{array}$ \\
\hline & $\begin{array}{l}k_{\mathrm{px}}=0.0 \\
k_{\mathrm{py}}=0.1 \times 10^{20} \\
k_{\mathrm{e} \theta}=0.1 \times 10^{20}\end{array}$ & $\begin{array}{l}k_{\mathrm{px}}=0.0 \\
k_{\mathrm{py}}=0.1 \times 10^{20} \\
k_{\mathrm{e} \theta}=0.1 \times 10^{20}\end{array}$ & $\begin{array}{l}k_{\mathrm{px}}=0.0 \\
k_{\mathrm{py}}=0.1 \times 10^{20} \\
k_{\mathrm{e} \theta}=0.1 \times 10^{20}\end{array}$ & $\begin{array}{l}k_{\mathrm{px}}=0.1 \times 10^{21} \\
k_{\mathrm{py}}=0.1 \times 10^{21} \\
k_{\mathrm{e} \theta}=0.1 \times 10^{-19}\end{array}$ \\
\hline & $F=170 \mathrm{kN}$ & $F=193 \mathrm{kN}$ & $F=193 \mathrm{kN}$ & $F=1 \times 10^{5} \mathrm{kN}$ \\
\hline
\end{tabular}

Table 4

Comparison between the analytical and numerical models

\begin{tabular}{lll}
\hline & Analytical model & Numerical model \\
\hline Spring elongation & $\Delta_{\mathrm{lt}}=0.1020 \times 10^{-3} \mathrm{~m}$ & $\Delta_{\mathrm{lt}}=0.1020 \times 10^{-3} \mathrm{~m}$ \\
& $\Delta_{\mathrm{lc}}=-0.5808 \times 10^{-3} \mathrm{~m}$ & $\Delta_{\mathrm{lc}}=-0.5808 \times 10^{-3} \mathrm{~m}$ \\
Bending moment & $M=35.8358 \mathrm{kN} \mathrm{m}$ & $M=35.8360 \mathrm{kN} \mathrm{m}$ \\
Rotation & $q_{1}=\phi=0.003239 \mathrm{rad}$ & $\phi^{(36)}=0.003239 \mathrm{rad}$ \\
Spring force & $F_{\mathrm{t}}=169.9 \mathrm{kN}$ & $F_{\mathrm{t}}=170.0 \mathrm{kN}$ \\
& $F_{\mathrm{c}}=-170.0 \mathrm{kN}$ & $F_{\mathrm{c}}=-170.0 \mathrm{kN}$ \\
Rotation centre & $x=0.03149 \mathrm{~m}$ & $x=0.03152 \mathrm{~m}$ \\
\hline
\end{tabular}

Comparative Graph - Analytical Model, Numerical Model and EC3

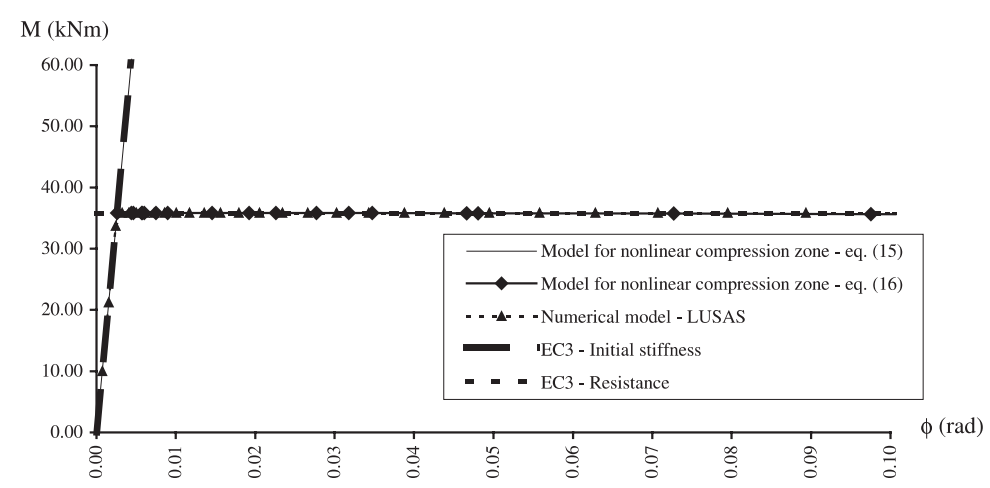

Fig. 11. Comparative graph.

that Eq. (24) does not apply in this case because the post-limit stiffness was chosen as zero.

Because experimental test results were available for this particular connection, it was possible to use them to calibrate the post-limit stiffness of the components, as well as adjusting the actual value of the moment resistance of the connection - Table 5 summarises the data. Using the same procedure as before, an initial calibration was performed with only the critical component with non-linear properties, illustrated in Fig. 13 with a 


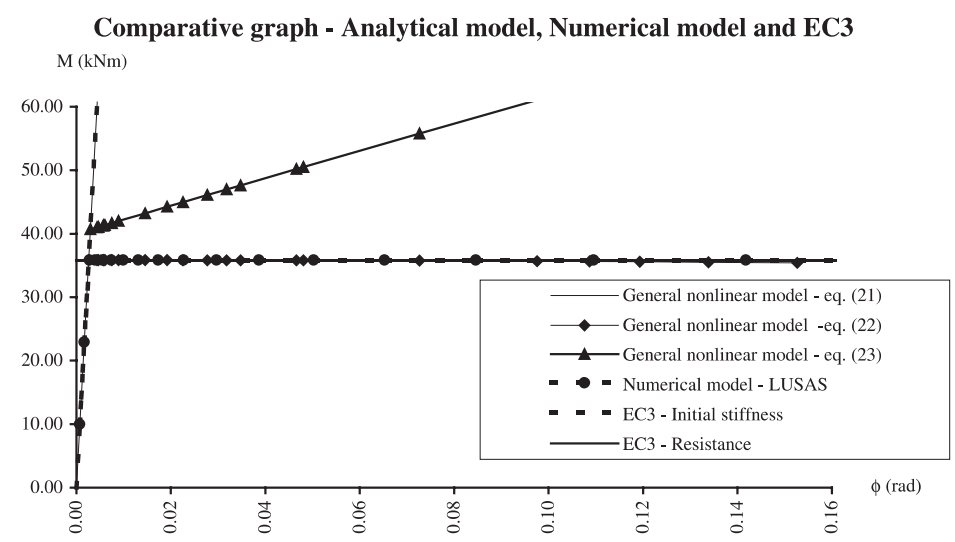

Fig. 12. Comparative graph.

Table 5

Data used in the general non-linear model of the connection

\begin{tabular}{ll}
\hline$k_{\mathrm{ec}}=3.82 \times 10^{5} \mathrm{kN} / \mathrm{m}$ & $k_{\mathrm{et}}=1.67 \times 10^{6} \mathrm{kN} / \mathrm{m}$ \\
$k_{\mathrm{pc}}=3.00 \times 10^{3} \mathrm{kN} / \mathrm{m}$ & $k_{\mathrm{pt}}=3.00 \times 10^{3} \mathrm{kN} / \mathrm{m}$ \\
$P_{\mathrm{C}}^{\mathrm{B}}=650 \mathrm{kN}$ & $P_{\mathrm{T}}^{\mathrm{B}}=795 \mathrm{kN}$ \\
$F=\frac{P_{\mathrm{C}}^{\mathrm{B}}}{2}=325 \mathrm{kN}$ & $F=\frac{P_{\mathrm{T}}^{\mathrm{B}}}{2}=397.5 \mathrm{kN}$ \\
\hline
\end{tabular}

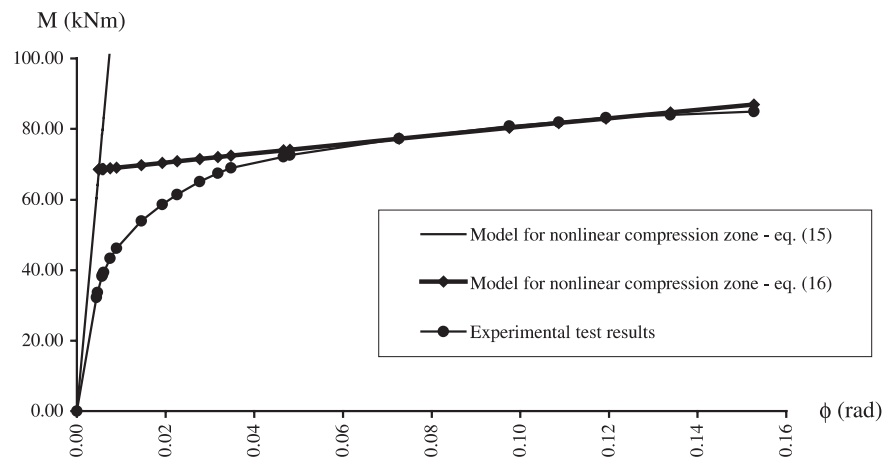

Fig. 13. Comparative graph.

subsequent full non-linear implementation, shown in Fig. 14.

\section{Concluding remarks}

The four degree-of-freedom elastic model presented in this paper was able to reproduce the full non-linear moment-rotation response of a steel connection. In particular, because of the post-buckling nature of the analysis, it was possible to pinpoint directly the bifur- cation points which correspond to the "yield" points of each component.

An accurate prediction of the post-limit response of the connection requires the knowledge of the post-limit stiffness $\left(k_{\mathrm{p}}\right)$ of each component. The analytical results presented here can very easily be used to calibrate the post-limit stiffness against experimental results, as was shown above.

Current work on the same model loaded in compression seems very promising, opening the way to the prediction of the behaviour of steel connections under combined loading (bending moment and axial force). 


\section{Comparative Graph - Analytical Model and Experimental Test Results}

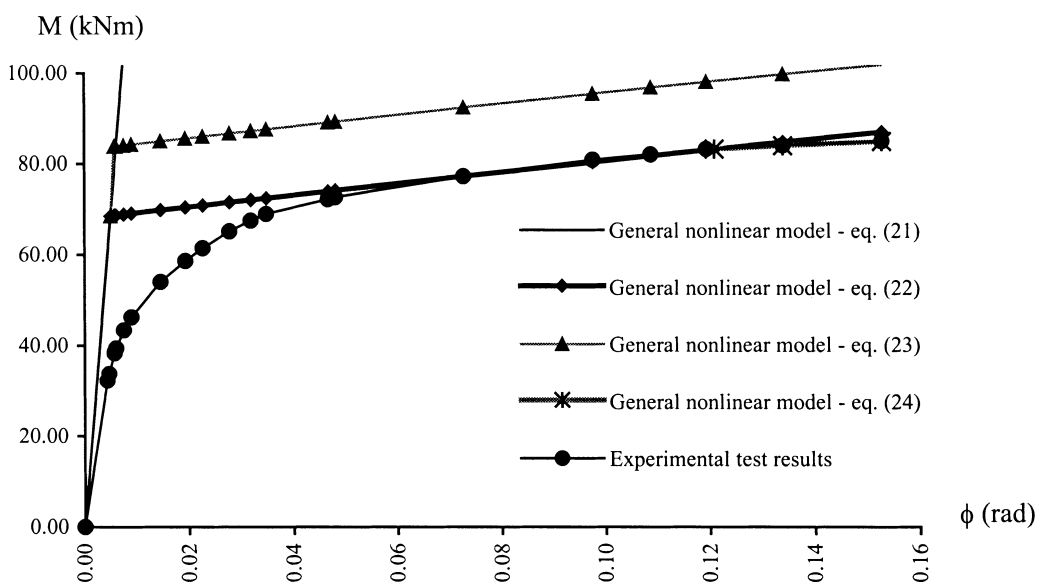

Fig. 14. Comparative graph.

\section{Acknowledgements}

The finantial support from the "Ministério da Ciência e Tecnologia" - PRAXIS XXI research project PRAXIS/P/ECM/13153/1998 and PRODEP II (Subprograma 1) is acknowledged.

\section{References}

[1] Weynand K, Jaspart JP, Steenhuis M. The stiffness model of revised Annex J of Eurocode 3. Proceedings of the Third International Workshop on Connections, 1995 May 8-31; Trento, Italy.

[2] Eurocode 3, ENV-1993-1-1, Revised Annex J, Design of Steel Structures, CEN, European Committee for Standardization, Document CEN/TC 250/SC 3 - N 419 E. Brussels, 1997.
[3] Simões da Silva L, Santiago A, Vila Real P. Ductility of steel connections. Canadian J Civil Engng, submitted for publication.

[4] Kuhlmann U. Influence of axial forces on the component web under compression. COST-C1 - Working Group Meeting, Thessaloniki, C1/WD2/99-01, May 28, 1998.

[5] Jaspart JP. Etude de la semi-rigidité des noeuds poutrecolonne et son influence sur la resistance des ossatures en acier. PhD Thesis, Department MSM, University of Liége, 1991.

[6] Shi YJ, Chan SL, Wong YL. Modelling for moment rotations characteristics for end-plate connections. J Struct Engng 1996;1300-6.

[7] Hunt GW, Burgan B. Hidden asymmetries in the Shanley model. J Mech Phys Solids 1985;33(1):83-94.

[8] Cruz PJS, Silva LAPS, Rodrigues DS, Simões RAD. Database for the semi-rigid behaviour of beam-to-column connections in seismic regions. J Construct Steel Res 1998;46(120):1-3.

[9] LUSAS User Manual. FEA Ltd, 1996. 